\title{
STABILITY OF THE MONOMIAL FUNCTIONAL EQUATION IN QUASI NORMED SPACES
}

\author{
Alireza Kamel Mirmostafaee
}

\begin{abstract}
Let $X$ be a linear space and $Y$ be a complete quasi $p$-norm space. We will show that for each function $f: X \rightarrow Y$, which satisfies the inequality$$
\left\|\Delta_{x}^{n} f(y)-n ! f(x)\right\| \leq \varphi(x, y)
$$

for suitable control function $\varphi$, there is a unique monomial function $M$ of degree $n$ which is a good approximation for $f$ in such a way that the continuity of $t \mapsto f(t x)$ and $t \mapsto \varphi(t x, t y)$ imply the continuity of $t \mapsto M(t x)$.
\end{abstract}

\section{Introduction}

The concept of stability of a functional equation arises when one replaces a functional equation by an inequality which acts as a perturbation of the equation. In 1940, Ulam [18] posed the first stability problem. In 1941, D. H. Hyers [10] gave the first significant partial solution to his question. Hyers' theorem was generalized for additive mappings by T. Aoki [3] in 1950 and D. G. Bourgin [5] in 1951. In 1978, Th. M. Rassias [17] solved the problem for linear mappings by considering an unbounded Cauchy difference. The phenomenon that was introduced and proved by Th. M. Rassias in the year 1978, is called the Hyers-Ulam-Rassias stability.

Let $X$ and $Y$ be linear spaces and $Y^{X}$ be the vector space of all functions from $X$ to $Y$. Following [11], for each $x \in X$, define $\Delta_{x}: Y^{X} \rightarrow Y^{X}$ by

$$
\Delta_{x} f(y)=f(x+y)-f(y), \quad f \in Y^{X}, y \in X .
$$

Inductively, we define

$$
\Delta_{x_{1}, \ldots, x_{n}} f(y)=\Delta_{x_{1}, \ldots, x_{n-1}}\left(\Delta_{x_{n}} f(y)\right)
$$

for each $x_{1}, \ldots, x_{n}, y \in X$ and $f \in Y^{X}$, we write

$$
\Delta_{x}^{n} f(y)=\Delta_{x_{1}, \ldots, x_{n}} f(y)
$$

Received February 17, 2009.

2000 Mathematics Subject Classification. Primary 39B52, 39B82; Secondary 47H10.

Key words and phrases. quasi $p$-norm, monomial functional equation, fixed point alternative, Hyers-Ulam-Rassias stability.

This research is supported by Ferdowsi University of Mashhad, No: MP88094MIM. 
if $x_{1}=\cdots=x_{n}=x$. By induction on $n$, it can be easily verified that

$$
\Delta_{x}^{n} f(y)=\sum_{k=0}^{n}(-1)^{n-k}\left(\begin{array}{l}
n \\
k
\end{array}\right) f(k x+y), \quad n \in \mathbb{N}, x, y \in X .
$$

It can be easily verified that every polynomial of degree at most $n$ satisfies the functional equation $\Delta_{x}^{n+1} f(y)=0$. Hence the functional equation $\Delta_{x}^{n} f(y)=0$ is called the polynomial functional equation of degree $n-1$. The functional equation

$$
\Delta_{x}^{n} f(y)=n ! f(x)
$$

is called the monomial functional equation of degree $n$, since the function $f(x)=c x^{n}$ is a solution of the functional equation. Every solution of the monomial functional equation of degree $n$ is said to be a monomial mapping of degree $n$. In particular additive, quadratic, cubic and quartic functions are monomials of degree one, two, three and four respectively.

The research on the stability of polynomial or monomial equations was initiated by D. H. Hyers in [11]. The problem has been recently considered by some authors see e.g. M. H. Albert and J. A. Baker [1], L. Cãdariu and V. Radu [6], A. Gilányi [8, 9], Z. Kaiser [13], Y.-H. Lee [14] and D. Wolna [19].

In Section 3, we use the fixed point alternative theorem to prove the HyersUlam-Rassias stability of monomial functional equation of an arbitrary degree in complete quasi-normed spaces. More precisely, we will show that if a function $f$ from a linear space $X$ to a complete $p$-normed space $Y$ for some $n \in \mathbb{N}$ satisfies the inequality

$$
\left\|\Delta_{x}^{n} f(y)-n ! f(x)\right\| \leq \varphi(x, y), \quad x, y \in X
$$

for suitable control function $\varphi$, then $f$ can be suitably approximated by a unique monomial $M: X \rightarrow Y$ of degree $n$. In Section 4, we will show that, for each $x \in X$, the continuity of $s \mapsto f(s x)$ and $s \mapsto \varphi(s x, s y)$ guarantee the continuity of $s \mapsto M(s x)$. It follows that in this case, $M(t x)=t^{n} M(x)$ for each $t \in \mathbb{R}$ and $x \in X$.

\section{Preliminaries}

In this section, we give some preliminaries, which will be used in this paper. We start by the following definition.

Definition 2.1. The pair $(X, d)$ is called a generalized complete metric space if $X$ is a nonempty set and $d: X^{2} \rightarrow[0, \infty]$ satisfies the following conditions:

(a) $d(x, y) \geq 0$ and the equality holds if and only if $x=y$,

(b) $d(x, y)=d(y, x)$,

(c) $d(x, z) \leq d(x, y)+d(y, z)$,

(d) every $d$-Cauchy sequence in $X$ is $d$-convergent.

Note that the distance between two points in a generalized metric space is permitted to be infinity. 
Definition 2.2. Let $(X, d)$ be a generalized complete metric space. A mapping $\Lambda: X \rightarrow X$ satisfies a Lipschitz condition with Lipschitz constant $L \geq 0$ if

$$
d(\Lambda(x), \Lambda(y)) \leq L d(x, y), \quad x, y \in X .
$$

If $L<1$, then $\Lambda$ is called a strictly contractive operator.

In 2003, Radu [15] employed the following result, due to Diaz and Margolis [7], to prove the stability of Cauchy additive functional equation. Using such an elegant idea, several authors applied the method to investigate the stability of some functional equations, see $[6,12,16]$.

Proposition 2.3 (The fixed point alternative principle). Suppose that a complete generalized metric space $(\mathcal{E}, d)$ and a strictly contractive mapping $J: \mathcal{E} \rightarrow \mathcal{E}$ with the Lipschitz constant $0<L<1$ are given. Then, for a given element $x \in \mathcal{E}$, exactly one of the following assertions is true: either

(a) $d\left(J^{n} x, J^{n+1} x\right)=\infty$ for all $n \geq 0$ or

(b) there exists some integer $k$ such that $d\left(J^{n} x, J^{n+1} x\right)<\infty$ for all $n \geq k$.

Actually, if (b) holds, then the sequence $\left\{J^{n} x\right\}$ is convergent to a fixed point $x^{*}$ of $J$ and

(b1) $x^{*}$ is the unique fixed point of $J$ in $\mathcal{F}:=\left\{y \in \mathcal{E}, d\left(J^{k} x, y\right)<\infty\right\}$;

(b2) $d\left(y, x^{*}\right) \leq \frac{d(y, J y)}{1-L}$ for all $y \in \mathcal{F}$.

Remark 2.4. The fixed point $x^{*}$, if it exists, is not necessarily unique in the whole space $\mathcal{E}$; it may depend on $x$. Actually, if (b) holds, then $(\mathcal{F}, d)$ is a complete metric space and $J(\mathcal{F}) \subset \mathcal{F}$. Therefore the properties (b1) and (b2) follow from "The Banach fixed point theorem".

Definition 2.5. A quasi-norm on a real vector space $X$ is a function $x \mapsto\||| x \mid\|$ from $X$ to $[0, \infty)$ which satisfies

(i) $\||| x \mid\|>0$ for every $x \neq 0$ in $X$,

(ii) $\||t x|\|=|t| \cdot|\|x \mid\|$ for every $t \in \mathbb{R}$ and $x \in X$,

(iii) there is $k \geq 1$ such that $\||x+y|\| \leq k(\||| x|||+\||y|\|)$ for every $x, y \in X$.

Aoki [2] (see also [4]) has shown that every quasi-normed space $(X,\||\cdot|\|)$ admits an equivalent quasi-norm $\|\cdot\|$ such that for some $0<p \leq 1$,

$$
\|x+y\|^{p} \leq\|x\|^{p}+\|y\|^{p}, \quad x, y \in X .
$$

In this case, $(X,\|\cdot\|)$ is called a quasi $p$-normed space. In special case, when $p=1,(X, \||\cdot|||)$ turns into a normed linear space.

\section{Stability of monomial functional equations}

Throughout the remainder of this paper, unless otherwise stated, we will assume that $0<p \leq 1$ and $q=\frac{1}{p}, X$ is a real vector space and $Y$ is a complete quasi $p$-norm space.

In 2006, D. Wolna in [19] proved the following result: 
Lemma 3.1. For every mapping $f: X \rightarrow Y$ and $m, k \in \mathbb{N}$, the following identity holds:

$$
f(2 x)-2^{m} f(x)=\frac{1}{m !}\left[\left(\begin{array}{c}
m \\
0
\end{array}\right) F_{1}(x)+\cdots+\left(\begin{array}{c}
m \\
m
\end{array}\right) F_{m+1}(x)-H(x)\right], \quad x \in X,
$$

where

$$
F_{i}(x)=\Delta_{x}^{m} f(i x)-m ! f(x), \quad x \in X ; i=1, \ldots, m+1
$$

and

$$
H(x)=\Delta_{2 x}^{m} f(x)-m ! f(2 x), \quad x \in X .
$$

Proof. [19, Lemma 2, page 102].

Corollary 3.2. If $f: X \rightarrow Y$ is a monomial of degree $m$, then

$$
f(2 x)=2^{m} f(x), \quad x \in X .
$$

Proof. If $f$ is a monomial of degree $m$, then $F_{1}(x)=\cdots=F_{m+1}(x)=H(x)=0$ for each $x \in X$. Hence the result follows from Lemma 3.1.

Lemma 3.3. Let $\psi: X \rightarrow[0, \infty)$ be a function and $\mathcal{E}=Y^{X}$. Define

$$
d(g, h)=\inf \left\{a>0:\|g(x)-h(x)\| \leq a^{q} \psi(x) \forall x \in X\right\}, \quad g, h \in \mathcal{E} .
$$

Then $d$ is a generalized complete metric on $\mathcal{E}$.

Proof. Clearly $d(f, g) \geq 0$ for each $f, g \in \mathcal{E}$ and the equality holds only when $f=g$. By the definition, $d$ is symmetric. Let $g, g^{\prime}, g^{\prime \prime} \in \mathcal{E}, d\left(g, g^{\prime}\right)<a_{1}$ and $d\left(g^{\prime}, g^{\prime \prime}\right)<a_{2}$. Then

$$
\left\|g(x)-g^{\prime}(x)\right\| \leq a_{1}^{q} \psi(x) \text { and }\left\|g^{\prime}(x)-g^{\prime \prime}(x)\right\| \leq a_{2}^{q} \psi(x)
$$

for each $x \in X$. It follows that

$$
\begin{aligned}
\left\|g(x)-g^{\prime \prime}(x)\right\|^{p} & \leq\left\|g(x)-g^{\prime}(x)\right\|^{p}+\left\|g^{\prime}(x)-g^{\prime \prime}(x)\right\|^{p} \\
& \leq\left(a_{1}+a_{2}\right)(\psi(x))^{p}, \quad x \in X .
\end{aligned}
$$

Therefore $d\left(g, g^{\prime \prime}\right) \leq a_{1}+a_{2}$. Since this holds for each $a_{1}, a_{2}$ with $d\left(g, g^{\prime}\right)<a_{1}$ and $d\left(g^{\prime}, g^{\prime \prime}\right)<a_{2}$,

$$
d\left(g, g^{\prime \prime}\right) \leq d\left(g, g^{\prime}\right)+d\left(g^{\prime}, g^{\prime \prime}\right)
$$

Let $\left\{f_{n}\right\}$ be a Cauchy sequence in $\mathcal{E}$. Then for each $\varepsilon>0$, there is some $n_{0} \in \mathbb{N}$ such that

$$
d\left(f_{n}, f_{m}\right)<\varepsilon, \quad n, m \geq n_{0} .
$$

By the definition, for each $n, m \geq n_{0}$,

$$
\left\|f_{n}(x)-f_{m}(x)\right\| \leq \varepsilon^{q} \psi(x), \quad x \in X .
$$

Hence, for each $x \in X,\left\{f_{n}(x)\right\}$ is a Cauchy sequence in complete space $Y$. It follows that $f(x)=\lim _{n \rightarrow \infty} f_{n}(x)$ exists for each $x \in X$. By (3.3) for each $n \geq n_{0}$,

$$
\left\|f(x)-f_{n}(x)\right\|=\lim _{m \rightarrow \infty}\left\|f_{m}(x)-f_{n}(x)\right\| \leq \varepsilon^{q} \psi(x), \quad x \in X .
$$


Therefore $d\left(f, f_{n}\right) \leq \varepsilon$ for each $n \geq n_{0}$. Hence $\lim _{n \rightarrow \infty} d\left(f, f_{n}\right)=0$. This completes the proof of the lemma.

Theorem 3.4. Let $\varphi: X \times X \rightarrow[0, \infty)$ and $f: X \rightarrow Y$ satisfy the inequality

$$
\left\|\Delta_{x}^{m} f(y)-m ! f(x)\right\| \leq \varphi(x, y), \quad x, y \in X
$$

for some $m \in \mathbb{N}$. Define

$$
\psi(x)=\frac{1}{2^{m} m !}\left(\sum_{i=0}^{m}\left[\left(\begin{array}{c}
m \\
i
\end{array}\right) \varphi(x,(i+1) x)\right]^{p}+[\varphi(2 x, x)]^{p}\right)^{q}, \quad x \in X .
$$

If for some $L<1$,

$$
2^{-m} \psi(2 x) \leq L \psi(x), \quad x \in X
$$

and $\lim _{n \rightarrow \infty} 2^{-m n} \varphi\left(2^{n} x, 2^{n} y\right)=0$ for all $x, y \in X$, then there exists a unique monomial mapping $M: X \rightarrow Y$ of degree $m$ such that

$$
\|M(x)-f(x)\| \leq \frac{\psi(x)}{\left(1-L^{p}\right)^{q}}, \quad x \in X .
$$

Proof. By Lemma 3.1

$$
\left\|2^{-m} f(2 x)-f(x)\right\| \leq \psi(x), \quad x \in X .
$$

Let $\mathcal{E}=Y^{X}$ and define

$$
d(g, h)=\inf \left\{a>0:\|g(x)-h(x)\| \leq a^{q} \psi(x), \forall x \in X\right\}, \quad g, h \in \mathcal{E} .
$$

By Lemma 3.3, $d$ is a complete generalized metric on $\mathcal{E}$. Define $J: \mathcal{E} \rightarrow \mathcal{E}$ by $J(g)(x)=2^{-m} g(2 x)$ for each $g \in \mathcal{E}$ and $x \in X$. Let $\varepsilon>0$ and $a=d(g, h)+\varepsilon$. Then by the definition,

$$
\|g(x)-h(x)\| \leq a^{q} \psi(x), \quad x \in X .
$$

Thanks to (3.6), for each $x \in X$,

$$
\begin{aligned}
\|J(g)(x)-J(h)(x)\| & =\left\|2^{-m} g(2 x)-2^{-m} h(2 x)\right\| \\
& \leq 2^{-m} a^{q} \psi(2 x) \\
& \leq L a^{q} \psi(x) .
\end{aligned}
$$

By the definition, $d(J(g), J(h)) \leq L^{p} a=L^{p}(d(g, h)+\varepsilon)$. Since $\varepsilon>0$ was arbitrary,

$$
d(J(g), J(h)) \leq L^{p} d(g, h), \quad g, h \in \mathcal{E} .
$$

This means that $J$ is a contractive mapping with Lipschitz constant $L^{p}<1$. By (3.8), $d(f, J(f)) \leq 1$, therefore, by Proposition 2.3, $J$ has a unique fixed point $M: X \rightarrow Y$ in the set $\mathcal{F}=\{g \in \mathcal{E}: d(f, g)<\infty\}$, where $M$ is defined by

$$
M(x):=\lim _{n \rightarrow \infty} J^{n}(f)(x)=\lim _{n \rightarrow \infty} 2^{-m n} f\left(2^{n} x\right), \quad x \in X .
$$

Moreover,

$$
d(f, M) \leq \frac{d(f, J(f))}{1-L^{p}} \leq \frac{1}{1-L^{p}}
$$


This means that (3.7) holds. Thanks to (1.1) and (3.9), for each $x, y \in X$, we have

$$
\begin{aligned}
& \left\|\Delta_{x}^{m} M(y)-m ! M(x)\right\| \\
= & \left\|\sum_{j=0}^{m}(-1)^{m-j}\left(\begin{array}{c}
m \\
j
\end{array}\right) M(j x+y)-m ! M(x)\right\| \\
= & \lim _{n \rightarrow \infty} 2^{-m n}\left\|\sum_{j=0}^{m}(-1)^{m-j}\left(\begin{array}{c}
m \\
j
\end{array}\right) f\left(2^{n} j x+2^{n} y\right)-m ! f\left(2^{n} x\right)\right\| \\
= & \lim _{n \rightarrow \infty} 2^{-m n}\left\|\Delta_{2^{n} x} f\left(2^{n} y\right)-m ! f\left(2^{n} x\right)\right\| \\
\leq & \lim _{n \rightarrow \infty} 2^{-m n} \varphi\left(2^{n} x, 2^{n} y\right)=0 .
\end{aligned}
$$

Hence $M$ is a monomial of degree $m$.

To prove the uniqueness assertion, let us assume that there exists a monomial $M^{\prime}: X \rightarrow Y$ of degree $m$, which satisfies (3.7). Thanks to Corollary $3.2, M^{\prime}$ is a fixed point of $J$ in $\mathcal{F}$. However, by Proposition $2.3, J$ has only one fixed point in $\mathcal{F}$, hence $M^{\prime} \equiv M$.

The following result can be obtained by imitating the proof of Theorem 3.4.

Theorem 3.5. With the notations of Theorem 3.4, let (3.4) hold for some $m \in \mathbb{N}$. If for some positive $L<1$,

$$
2^{m} \psi\left(2^{-1} x\right) \leq L \psi(x), \quad x \in X
$$

and $\lim _{n \rightarrow \infty} 2^{m n} \varphi\left(2^{-n} x, 2^{-n} y\right)=0$. Then there exists a unique monomial $M: X \rightarrow Y$ of degree $m$ such that

$$
\|M(x)-f(x)\| \leq \frac{L \psi(x)}{\left(1-L^{p}\right)^{q}}, \quad x \in X .
$$

\section{Continuity behavior of monomial mappings}

In this section, we investigate continuity of monomial mappings in quasi $p$ normed spaces. In fact, we will show that under some conditions on $f$ and $\psi$, the monomial mapping $s \longmapsto M(s x)$ is continuous.

Theorem 4.1. Let the conditions of theorem 3.4 (or Theorem 3.5) hold. If for each $x \in X$, the function $s \mapsto f(s x)$ from $\mathbb{R}$ to $Y$ is continuous at some point $s_{0}$ and for some $x_{0} \in X$, the function $s \mapsto \psi\left(s x_{0}\right)$ from $\mathbb{R}$ to $[0, \infty)$ is bounded in a neighborhood of $s_{0}$, then $s \longmapsto M\left(s x_{0}\right)$ from $\mathbb{R}$ to $Y$ is continuous at $s_{0}$.

Proof. We prove the theorem under conditions of Theorem 3.4. The proof for the other case is similar. Let $\alpha>0$ and $\delta_{1}>0$ be such that

$$
\left|s-s_{0}\right|<\delta_{1} \Rightarrow \psi\left(s x_{0}\right)<\alpha .
$$


Given $\varepsilon>0$, choose some $n_{0}$ so that

$$
\frac{L^{n_{0}} \alpha}{\left(1-L^{p}\right)^{q}}<\frac{\varepsilon}{3^{q}}
$$

Since $0<L<1$, such a choice is possible. By the continuity of $s \mapsto f\left(2^{n_{0}} s x_{0}\right)$, we can find positive $\delta \leq \delta_{1}$ such that

$$
\left|s-s_{0}\right|<\delta \Rightarrow\left\|f\left(2^{n_{0}} s x_{0}\right)-f\left(2^{n_{0}} s_{0} x_{0}\right)\right\|<\frac{\varepsilon}{3^{q} 2^{-m n_{0}}} .
$$

Let $\left|s-s_{0}\right|<\delta$. Then

$$
\begin{aligned}
& \left\|M\left(s x_{0}\right)-M\left(s_{0} x_{0}\right)\right\|^{p} \\
= & \left\|2^{-m n_{0}} M\left(2^{n_{0}} s x_{0}\right)-2^{-m n_{0}} M\left(2^{n_{0}} s_{0} x_{0}\right)\right\|^{p} \\
\leq & 2^{-m n_{0} p}\left(\left\|M\left(2^{n_{0}} s x_{0}\right)-f\left(2^{n_{0}} s x_{0}\right)\right\|^{p}+\left\|f\left(2^{n_{0}} s x_{0}\right)-f\left(2^{n_{0}} s_{0} x_{0}\right)\right\|^{p}\right. \\
& \left.\quad+\left\|f\left(2^{n_{0}} s_{0} x_{0}\right)-M\left(2^{n_{0}} s_{0} x_{0}\right)\right\|^{p}\right) \\
< & \frac{\varepsilon^{p}}{3}+\frac{\varepsilon^{p}}{3}+\frac{\varepsilon^{p}}{3}=\varepsilon^{p} .
\end{aligned}
$$

That is

$$
\left|s-s_{0}\right|<\delta \Rightarrow\left\|M\left(s x_{0}\right)-M\left(s_{0} x_{0}\right)\right\|<\varepsilon .
$$

The above inequality proves continuity of $s \mapsto M\left(s x_{0}\right)$ at $s_{0}$.

Corollary 4.2. If $s \mapsto f(s x)$ and $s \mapsto \psi(s x)$ from $\mathbb{R}$ to $Y$ and $\mathbb{R}^{+}$respectively are continuous, then $s \mapsto M(s x)$ is continuous. In this case, $M(t x)=t^{m} M(x)$ for each $t \in \mathbb{R}$ and $x \in X$.

Proof. Follows from Theorem 4.1 and [13, Lemma 5].

Corollary 4.3. Let $(X,\||\cdot|\|)$ be a normed space. Let for some $\varepsilon>0$ and positive real number $\beta \neq m, f: X \rightarrow Y$ satisfy the inequality

$$
\left\|\Delta_{x}^{m} f(y)-m ! f(x)\right\| \leq \varepsilon\left(\left\|\left|x\|\|^{\beta}+\|\mid y\|^{\beta}\right), \quad x, y \in X .\right.\right.
$$

(i) If $\beta<m$, there is a unique monomial $M: X \rightarrow Y$ of degree $m$ such that

$$
\begin{aligned}
& \|f(x)-M(x)\| \\
\leq & \frac{\varepsilon\|x\| \|^{\beta}}{2^{m} m !\left(1-2^{(\beta p-m p)}\right)^{q}}\left(\sum_{i=0}^{m}\left[\left(\begin{array}{c}
m \\
i
\end{array}\right)\left(1+(i+1)^{\beta}\right)\right]^{p}+\left(2^{\beta}+1\right)^{p}\right)^{q}, x \in X .
\end{aligned}
$$

(ii) If $\beta>m$, there is a unique monomial $M: X \rightarrow Y$ of degree $m$ such that

$$
\begin{aligned}
& \|f(x)-M(x)\| \\
\leq & \frac{\varepsilon 2^{(m-\beta)}\|x\|^{\beta}}{2^{m} m !\left(1-2^{(m p-\beta p)}\right)^{q}}\left(\sum_{i=0}^{m}\left[\left(\begin{array}{c}
m \\
i
\end{array}\right)\left(1+(i+1)^{\beta}\right)\right]^{p}+\left(2^{\beta}+1\right)^{p}\right)^{q}, x \in X .
\end{aligned}
$$


Furthermore, if $f$ is continuous, then $M$ is continuous and $M(t x)=t^{m} M(x)$ for each $t \in \mathbb{R}$ and $x \in X$.

Proof. Let $\varphi(x, y)=\varepsilon\left(\|\| x\left|\|\|^{\beta}+\||| y\|^{\beta}\right)\right.$ and $\psi$ be defined by (3.5). Then

$$
\psi(x)=\frac{\varepsilon\left\|\left|\|x \mid\|^{\beta}\right.\right.}{2^{m} m !}\left(\sum_{i=0}^{m}\left[\left(\begin{array}{c}
m \\
i
\end{array}\right)\left(1+(i+1)^{\beta}\right)\right]^{p}+\left(2^{\beta}+1\right)^{p}\right)^{q}, x \in X .
$$

If $\beta<m$, then

$$
2^{-m} \psi(2 x)=2^{-m+\beta} \psi(x), x \in X .
$$

Hence for $L=2^{-m+\beta}<1$, the conditions of Theorem 3.4 hold. Therefore, we can find a unique monomial function of degree $m$ such that

$$
\|f(x)-M(x)\| \leq \frac{\psi(x)}{\left(1-2^{(\beta p-m p)}\right)^{q}}, x \in X .
$$

This proves (i). In case (ii), when $\beta>m$, by (4.2), we can write

$$
2^{m} \psi\left(2^{-1} x\right)=2^{m-\beta} \psi(x), x \in X .
$$

By Theorem 3.5 for $L=2^{m-\beta}$, there is a unique monomial function $M: X \rightarrow Y$ of degree $m$ such that

$$
\|f(x)-M(x)\| \leq \frac{2^{(m-\beta)} \psi(x)}{\left(1-2^{(m p-\beta p)}\right)^{q}}, x \in X .
$$

The last assertion follows from Theorem 4.1.

Corollary 4.4. Let for some $\varepsilon>0, f: X \rightarrow Y$ satisfy the inequality

$$
\left\|\Delta_{x}^{m} f(y)-m ! f(x)\right\| \leq \varepsilon, \quad x, y \in X .
$$

Then there is a unique continuous monomial mapping $M: X \rightarrow Y$ of degree $m$ such that

$$
\|f(x)-M(x)\| \leq \frac{\varepsilon\left(\sum_{i=0}^{m}\left(\begin{array}{c}
m \\
i
\end{array}\right)^{p}+1\right)^{q}}{\left(2^{m} m !\right)\left(1-2^{-m p}\right)^{q}}, \quad x \in X .
$$

Furthermore if $f$ is continuous, then $M$ is continuous and $M(t x)=t^{m} M(x)$ for each $t \in \mathbb{R}$ and $x \in X$.

Proof. Apply Theorem 3.4 for $L=2^{-m}$ and $\varphi(x, y)=\varepsilon, \quad x, y \in X$.

\section{References}

[1] M. Albert and J. A. Baker, Functions with bounded nth differences, Ann. Polon. Math. 43 (1983), no. 1, 93-103.

[2] T. Aoki, Locally bounded linear topological spaces, Proc. Imp. Acad. Tokyo 18 (1942), 588-594.

[3], On the stability of the linear transformation in Banach spaces, J. Math. Soc. Japan 2 (1950), 64-66.

[4] Y. Benyamini and J. Lindenstrauss, Geometric Nonlinear Functional Analysis. Vol. 1, American Mathematical Society Colloquium Publications, 48. American Mathematical Society, Providence, RI, 2000. 
[5] D. G. Bourgin, Classes of transformations and bordering transformations, Bull. Amer. Math. Soc. 57 (1951), 223-237.

[6] L. Cădariu and V. Radu, Remarks on the stability of monomial functional equations, Fixed Point Theory 8 (2007), no. 2, 201-218.

[7] J. B. Diaz and B. Margolis, A fixed point theorem of the alternative, for contractions on a generalized complete metric space, Bull. Amer. Math. Soc. 74 (1968), 305-309.

[8] A. Gilányi, Hyers-Ulam stability of monomial functional equations on a general domain, Proc. Natl. Acad. Sci. USA 96 (1999), no. 19, 10588-10590.

[9] _ On the stability of monomial functional equations, Publ. Math. Debrecen 56 (2000), no. 1-2, 201-212.

[10] D. H. Hyers, On the stability of the linear functional equation, Proc. Natl. Acad. Sci. USA 27 (1941), 222-224.

[11] _ Transformations with bounded mth differences, Pacific J. Math. 11 (1961), 591-602.

[12] S.-M. Jung, T.-S. Kim, and K.-S. Lee, A fixed point approach to the stability of quadratic functional equation, Bull. Korean Math. Soc. 43 (2006), no. 3, 531-541.

[13] Z. Kaiser, On stability of the monomial functional equation in normed spaces over fields with valuation, J. Math. Anal. Appl. 322 (2006), no. 2, 1188-1198.

[14] Y.-H. Lee, On the stability of the monomial functional equation, Bull. Korean Math. Soc. 45 (2008), no. 2, 397-403.

[15] V. Radu, The fixed point alternative and the stability of functional equations, Fixed Point Theory 4 (2003), no. 1, 91-96.

[16] J. M. Rassias, Alternative contraction principle and Ulam stability problem, Math. Sci. Res. J. 9 (2005), no. 7, 190-199.

[17] Th. M. Rassias, On the stability of the linear mapping in Banach spaces, Proc. Amer. Math. Soc. 72 (1978), no. 2, 297-300.

[18] S. M. Ulam, Problems in Modern Mathematics, Science Editions John Wiley \& Sons, Inc., New York 1964.

[19] D. Wolna, The stability of monomial functions on a restricted domain, Aequationes Math. 72 (2006), no. 1-2, 100-109.

Department of Pure Mathematics

Center of Excellence in Analysis on Algebraic Structures

FERDOWSi UNIVERSiTy OF MASHHAD

P.O. 1159, MASHHAD 91775, IRAN

E-mail address: mirmostafaei@ferdowsi.um.ac.ir 